\section{Comparação de métodos de regressão multivariada no estudo de determinantes da cárie dentária em crianças}

\section{Comparison of multivariate regression methods in the study of determining factors for tooth decay in children}

Márcia Maria Dantas Cabral de Melo 1

Wayner Vieira de Souza 2

Geraldo Bosco Lindoso Couto 3

\begin{abstract}
Objectives: to compare the results of hierarchical and conventional multivariate models in the investigation of determining factors for tooth decay in children.

Methods: an analysis was conducted of an investigation of children served by the Family Health Strategy in Recife, Pernambuco, carried out 2006. The dependent variable was the prevalence of tooth decay (ceo-d $\geq 1$ ); The independent variables were ordered in blocks: socio-economic, lifelfamily context, dental health care. Variables statistically associated with ceo- $d \geq 1$ in univariate analyses with a level of significance of $p \leq 0.20$ were included in the multivariate models. In the hierarchical mode, the order of inclusion of variables was guided by a multidimensional conceptual model. Associated variables with a level of significance of $p \leq 0.10$ were retained in the final model. In the conventional logistic model, the significant variables from the univariate analyses were simultaneously included and adjusted. Variables associated with ceo-d with $p \leq 0.10$ were retained.

Results: in the conventional model the factors associated with ceo-d $d \geq 1$ were high household density, low level of education of caregiver; studying at public school, seeking treatment/emergency services, and eating sweets. In the hierarchical model, the occupation of the father and the level of education of the mother were also retained in the final model.

Conclusions: hierarchical analysis enabled the identification of distal determining factors, which influence lower level factors and their influence on the outcome, thereby providing a more contextualized discussion of the issue.
\end{abstract}

Key words Dental caries, Child, Social conditions, Health services, Multivariate analysis
1 Departamento de Clínica e Odontologia Preventiva. Universidade Federal de Pernambuco. Av. Professor Moraes Rego, s.n. Cidade Universitária. Recife, PE, Brasil. CEP. 50.670-420.

E-mail: marciamdcm@hotmail.com

2 Centro de Pesquisa Aggeu Magalhães. Fundação Oswaldo Cruz. Recife, PE, Brasil.

3 Programa de Pós-Graduação de Odontologia. Universidade Federal de Pernambuco. Recife, PE, Brasil.

\section{Resumo}

Objetivos: comparar resultados com modelos multivariados hierarquizados e convencionais na investigação de determinantes da cárie dentária em crianças.

Métodos: analisou-se dados de um inquérito em crianças assistidas pela Estratégia Saúde da Família do Recife, Pernambuco, conduzido em 2006. A variável dependente foi a prevalência de cárie (ceo$d \geq 1)$; As variáveis independentes foram ordenadas em blocos: socioeconômico, contexto de vida/familiar, cuidado em saúde bucal. Variáveis estatisticamente associadas a ceo- $d \geq 1$ em análises univariadas, com um nivel de significância de $p \leq 0,20$, foram incluidas nos modelos multivariados. No modelo hierarquizado, a ordem de inclusão das variáveis foi orientada por um modelo conceitual multidimensional. Variáveis associadas a um nivel de $p \leq 0,10$ foram mantidas no modelo final. No modelo logístico convencional as variáveis significantes nas análises univariadas foram simultaneamente incluidas e ajustadas. Variáveis associadas ao ceo-d com $p \leq 0,10$ foram mantidas.

Resultados: no modelo convencional foram fatores associados ao ceo- $d \geq 1$ : alta densidade domiciliar, baixa escolaridade do cuidador; estudar em escolas públicas, procurar tratamento/emergência, ingerir doces. No modelo hierarquizado, além desses fatores, foram mantidos no modelo final a ocupação do pai e a escolaridade da mãe.

Conclusões: a análise hierarquizada possibilitou identificar os determinantes distais, que exerceram influências sobre os de niveis inferiores e destes sobre o desfecho, permitindo uma discussão mais contextualizada do problema.

Palavras-chave Cárie dentária, Criança, Condições sociais, Serviços de saúde, Análise multivariada 


\section{Introdução}

A despeito das melhorias na experiência de cárie dentária observadas internacionalmente em préescolares, a ocorrência desse agravo ainda se mantém elevada e com padrão de distribuição heterogêneo. ${ }^{1}$ No Brasil, apesar dos avanços recentes nas políticas públicas de saúde bucal, 2 o último levantamento nacional realizado em 2010 revelou uma tímida melhora dos indicadores de cárie para as crianças com cinco anos de idade. ${ }^{3}$ Esses dados sugerem que os esforços nacionais empreendidos na promoção da saúde bucal ampla e universal não estão sendo suficientes para reduzir a ocorrência de cárie na população pré-escolar a níveis considerados satisfatórios. 2,4

Entre os métodos de análise de dados utilizados na identificação de fatores determinantes da cárie dentária, os mais usuais são modelos de regressão multivariados, nos quais as variáveis explanatórias são tratadas como se situassem em um único nível hierárquico de determinação, sem se considerar os diferentes níveis de explicação dos determinantes da cárie no processo saúde-doença. 5

Buscando-se obter parâmetros mais próximos da realidade, tem-se lançado mão de abordagens teóricas e analíticas mais complexas, como aquelas que consideram a hierarquia, a temporalidade ou inter-relação entre as variáveis independentes $5,6 \mathrm{e}$ igualmente, as relações entre os diferentes níveis de determinação e natureza dos fatores que atuam na produção da cárie. Assim, diversos estudos têm investigado os fatores relacionados à saúde bucal utilizando abordagens analíticas multivariadas ancoradas em modelos conceituais subjacentes às teorias da determinação social da saúde, como os modelos de análise multinível.7-10

Essas análises têm como vantagem a possibilidade de se incorporar os efeitos e interações dos fatores relacionados às condições gerais de vida, como a estrutura social e econômica; ao contexto social local, das políticas públicas de promoção à saúde e do acesso aos serviços de saúde e os individuais, vinculados à biologia humana e ao comportamento relacionado à saúde. 9,10

Victora et al.11 propõem, para estudos cuja unidade de observação é o indivíduo, a utilização de modelos de análise cujas variáveis explicativas são organizadas em distintos níveis hierárquicos e orientados por um modelo teórico correspondente, e não apenas na presença de associações estatísticas. 11-12 Essa estratégia busca reduzir as distorções na estimação dos efeitos dos determinantes distais (socioeconômicos), possibilitando a interpretação dos resul- tados de forma mais ampliada. Nessa abordagem, o pressuposto de que as observações não são independentes e estão ligadas a níveis hierarquizados, significa a violação de independência da análise multivariada convencional. 12

No campo da saúde bucal infantil, tais metodologias têm sido aplicadas quando há interesse em se analisar o efeito dos múltiplos determinantes, em seus distintos níveis de atuação, envolvidos na produção da cárie. ${ }^{5,13}$ No Brasil, estas abordagens analíticas, como as hierarquizadas ${ }^{14-16}$ e multinível, ${ }^{9}$ têm sido utilizadas na investigação dos múltiplos determinantes para cárie em pré-escolares, entretanto, no âmbito da atenção primária à saúde, esses estudos ainda são escassos, ${ }^{9,10}$ assim como aqueles de abordagem convencional.17,18

No Recife, um inquérito de cárie na dentição decídua de 2.020 pré-escolares com idades entre 18 e 36 meses e com cinco anos, em 2006, conduzido em unidades básicas de saúde, revelou elevadas prevalências de cárie nesta população. 19 Fatores socioambientais e comportamentais associados à cárie nesta população foram investigados utilizando abordagem analítica do tipo caso-controle e modelos de análise multivariados convencionais. 20 Ao final, constatou-se que os principais fatores associados à cárie foram aqueles relacionados ao contexto social. 20

O presente artigo compara os resultados das análises dos fatores de risco para a cárie dentária na população dos estudos anteriormente citados, 19,20 com a aplicação de modelos de regressão logística multivariado hierarquizado e convencional. Para evidenciar o poder explicativo dos dois modelos sobre o papel dos diferentes fatores na produção da cárie infantil.

\section{Métodos}

Utilizou-se o banco de dados do inquérito de prevalência de cárie dentária na dentição decídua realizado em 2006 em crianças com cinco anos de idade cadastradas nas unidades de saúde (USF) da Estratégia Saúde da Família (ESF) dos Distritos Sanitários (DS) II e IV, na cidade do Recife.19,20 Informações sobre a confiabilidade e validade do inquérito estão disponíveis em publicações anteriores. 19,20

O tamanho da amostra do inquérito foi calculado admitindo-se uma prevalência de cárie de $65 \%$ para a idade de cinco anos, com base nos dados levantamento nacional de 2002-2003 para a região Nordeste, erro amostral de $6,5 \%$, grau de confiança de $95 \%$ e efeito de desenho igual a, um, obtendo-se 
uma amostra de 1.000 crianças para o conjunto de crianças dos dois DS. 19

A alocação da amostra em cada DS foi realizada por cotas iguais de crianças $(n=600$ em cada distrito). Assim, 9.480 famílias foram selecionadas proporcionalmente ao número de famílias cadastradas nas USF de cada DS. Em seguida, incluíram-se todas as crianças com idade prevista pelo estudo, 19 recrutadas nos domicílios. A coleta de dados foi realizada nas USFs nas quais as crianças estavam cadastradas. Um total de 605 crianças com cinco anos de idade residentes nos dois DS participaram do estudo, obtendo-se uma taxa global de participação de $50,4 \%$. Ao final, a prevalência de cárie dentária ponderada e seus respectivos IC de $95 \%$, nesta população foi de $63,8 \%(59,8-67,6) .19$

Os fatores de risco relacionados à cárie foram, inicialmente, investigados em um estudo caso-controle aninhado ao inquérito referido no qual se utilizou uma abordagem analítica convencional, não hierarquizada. 20 As variáveis independentes foram agrupadas em quatro blocos (sociodemográficas e ambientais, estrutura familiar, cuidados com a saúde, uso de serviços de educação e saúde), tendo a associação com o desfecho sido analisada em duas etapas (realização de ajuste do modelo internamente nos blocos e no modelo final). Isto é, as variáveis que apresentaram associação estatisticamente significante com o desfecho nos modelos de regressão múltipla, dentro de cada bloco, foram reunidas em um novo modelo multivariado para obtenção do modelo final. 20

O processo de amostragem para o referido estudo foi realizado buscando-se reproduzir as estimativas de prevalência encontradas no inquérito, além de restituir a correta proporcionalidade entre o número de indivíduos examinados e o número de famílias cadastradas em cada DS. Para tanto, obtevese uma amostra aleatória simples de casos e controles da população com cinco anos de idade que participou do inquérito, um total de 374 casos (62\%) e 231 controles.

A definição de casos (índice ceo-d $\geq 1$ : número de dentes cariados, perdidos e obturados na dentição decídua maior ou igual a um) e controles (índice ceo-d igual a zero) foi semelhante nas duas análises: convencional, publicada previamente 20 e hierarquizada.

As variáveis explicativas utilizadas em ambas as abordagens analíticas foram: a) variáveis socioeconômicas (número de moradores por domicílio, número de pessoas por quarto de dormir, escolaridade materna, ocupação do pai, ocupação da mãe, escolaridade do cuidador); b) relacionadas ao contexto de vida e familiar (fatores do ambiente de moradia: tempo de moradia na área, abastecimento de água pela rede geral, origem da água do domicílio, canalização interna, distrito sanitário de moradia; fatores de suporte social: frequentar préescola, tipo de pré-escola, utilização das ações de saúde bucal nas USF, razão da procura às USF (educação, prevenção, tratamento, emergência), utilização de outros serviços de saúde bucal; fatores da estrutura familiar: cuidador diário da criança, número de irmãos que moram juntos, com quem a criança mora, expediente de trabalho da mãe, ordem de nascimento) e c) atitudes de cuidado em saúde bucal (consumo de doces entre as refeições, dados sobre a escovação (realização, frequência diária, uso de pasta dental, quem a realiza e sexo).

$\mathrm{Na}$ análise de regressão múltipla hierarquizada, as variáveis independentes foram organizadas em blocos de acordo com um modelo explicativo hierarquizado para a cárie dentária, tendo por base modelos teóricos sobre os determinantes sociais e biológicos da saúde 7,8 e do agravo em questão, 6 conforme apresentado na Figura 1. Este modelo adotou as variáveis socioeconômicas como os principais fatores determinantes na intermediação das demais variáveis relacionadas ao desfecho em questão.

$\mathrm{Na}$ análise de regressão logística multivariada convencional, foram incluídas no modelo todas as variáveis independentes que estiveram estatisticamente associadas ao evento na análise univariada $(p \leq 0,20)$, ajustadas conjuntamente, sem considerar ordem hierárquica ou nível de determinação. Foram mantidas no modelo final as variáveis que apresentaram $p \leq 0,10$.

Em ambos os modelos de análise, inicialmente estimou-se a magnitude do efeito de cada variável calculando-se a Odds ratio (OR) bruta e seus respectivos Intervalos de Confiança (IC) a 95\%. Aquelas variáveis que apresentaram associação com ceo- $\mathrm{d} \geq 1$ a um nível de significância de $p \leq 0,20$ foram incluídas nos modelos de regressão logística multivariados (hierarquizado e convencional). O método backward stepwise foi utilizado na seleção das variáveis para a análise multivariada, com base no valor do logaritmo da razão de verossimilhança. Os passos das análises de regressão logística multivariada, que foi conduzida de acordo com os procedimentos propostos por Victora et al.,11 são descritos a seguir:

Inicialmente as variáveis estatisticamente associadas ao desfecho na análise univariada $(p \leq 0,20)$ foram incluídas nos modelos multivariados correspondentes a seu nível de determinação (distal, 
Figura 1

Modelo explicativo hierarquizado dos potenciais fatores associados à prevalência de cárie dentária nas crianças com cinco anos de idade.

$1^{\circ}$ Nível de determinação

(Fatores distais)

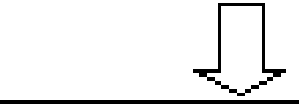

Bloco de variáveis socioeconômicas

número de moradores por domicílio, número de pessoas por quarto de dormir, escolaridade materna, ocupação do pai, ocupação da mãe, escolaridade do cuidador.

$2^{\circ}$ Nível de determinação

(Fatores intermediários)

Bloco de variáveis do contexto de vida e familiar

Do ambiente de moradia: tempo de moradia na área, fornecimento de água pela rede geral, origem da água do domicílio,canalização interna, distrito sanitário de moradia.

Do suporte social: frequentar pré-escola, tipo de pré-escola, utilização das ações de saúde bucal nas USF, razão da procura às USF, utilização de outros serviços de saúde bucal.

Da estrutura familiar: cuidador diário da criança, número de irmãos que moram juntos, com quem a criança mora, expediente de trabalho da mãe, ordem de nascimento.

$3^{\circ}$ Nível de determinação

(Fatores proximais)

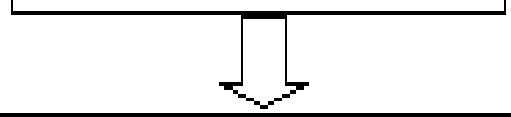

Bloco de variáveis relativas a atitudes de cuidado

Consumo de doces entre as refeições, realizar escovação, frequência diária de escovação, uso de pasta dental nas escovações, quem realiza a escovação, sexo. 
intermediário e proximal). Foram mantidas no modelo final de cada bloco, as variáveis associadas ao desfecho com nível de $p \leq 0,10$. Nas análises multivariada hierarquizada, as variáveis do bloco socioeconômico ( $1^{\circ}$ nível de determinação) que se mantiveram independentemente associadas $(p \leq 0,10)$ ao evento foram as primeiras a serem incluídas no modelo, permanecendo como fatores de ajuste para as variáveis posicionadas hierarquicamente nos níveis inferiores (Figura 1). Inicialmente, se juntaram a elas aquelas variáveis de $2^{\circ}$ nível de determinação (fatores intermediários) com $p \leq 0,10$, gerando um novo modelo multivariado. Nesse modelo foram excluídas apenas às variáveis de $2^{\circ}$ nível com $p>0,10$, pois segundo os critérios seguidos para essa análise hierarquizada, 11 as variáveis de $1^{\circ}$ nível de determinação distal permanecem independentemente do valor de $p$ considerado $(p \leq 0,10)$. Em seguida, da mesma forma, as variáveis de $3^{\circ}$ nível de determinação (fatores proximais) se juntaram às de $1^{\circ}$ nível e às de $2^{\circ}$ nível que se mantiveram com $p \leq 0,10$. O critério de permanência das variáveis no modelo hierarquizado final foi de $p \leq 0,10$, isto é, para as variáveis de nível intermediário e proximal.

Todas as análises foram realizadas utilizando o programa estatístico Statistical Package for the Social Sciences (SPSS) na versão 8.0. O projeto de pesquisa foi aprovado pelo Comitê de Ética em Pesquisas do Centro de Ciências da Saúde da Universidade Federal de Pernambuco (CEP/CCS/UFPE), sob o número 118/11.

\section{Resultados}

Um total de 479 participantes foi incluído, sendo $301(62,8 \%)$ crianças com ceo-d $\geq 1$ e 178 com ceo$\mathrm{d}=0.20$.

Os resultados das análises univariadas mostraram para o grupo das variáveis socioeconômicas, que apenas a ocupação da mãe $(p=0,381)$ não esteve estatisticamente associada a ceo- $d \geq 1$. Quanto às 15 variáveis relativas ao contexto de vida e familiar, oito delas não atingiram um nível de significância de $p \leq 0,20$ (fornecimento de água pela rede geral: $p=0,224$; origem da água do domicílio: $p=0,970$; canalização interna: $p=0,705$; distrito sanitário de moradia: $p=0,776$; frequentar préescola: $p=0,431$; razão da procura às USF (educativa/orientação: $p=0,608$; prevenção: $p=0,460$ ); cuidador diário da criança: $p=0,704$; com quem a criança mora: $p=0,645)$. Em relação às variáveis concernentes a atitudes de cuidados, apenas a variável sexo $(p=0,572)$ não esteve positivamente associada com ceo- $\mathrm{d} \geq 1$.

A Tabela 1 apresenta os resultados da análise de regressão logística multivariada hierarquizada (OR ajustada 2). Observam-se os valores das ORs brutas obtidos nas análises univariadas e ajustadas de cada bloco (OR ajustada1).

No nível distal de determinação são apresentadas as variáveis que foram incluídas e ajustadas no modelo bloco socioeconômico $\left(\mathrm{OR}_{\text {ajustada1 } 1}\right)$. As variáveis deste bloco que atingiram uma significância de $p \leq 0,10$, foram incluídas para um novo ajuste $\left(\mathrm{OR}_{\text {ajustada2 }}\right)$. Todas estas variáveis (número de moradores por domicílio, $p=0,005$, escolaridade materna, $p=0,182$, ocupação do pai, $p=0,126$, escolaridade do cuidador, $p=0,244$ ) foram mantidas neste bloco, independente de apresentarem ou não significância estatística, por se tratarem de fatores de ajuste para as variáveis hierarquicamente inferiores, como preconizado por Victora et al.11 Apenas o número de moradores por domicílio permaneceu no modelo final $(p \leq 0,10)$. No nível intermediário, permaneceram associados positivamente a ceo- $d \geq 1(p \leq 0,10)$ duas variáveis contextuais relacionadas ao suporte social: o tipo de escola (pública ou filantrópica) e a busca por serviços de saúde bucal para tratamento ambulatorial ou de emergência. No nível proximal, a variável relativa ao padrão de ingestão de doces permaneceu fortemente associada, mesmo após o ajuste pelos blocos superiores.

A Tabela 2 apresenta os resultados finais da análise de regressão logística multivariada convencional. Dentre as 19 variáveis que obtiveram uma significância de $p \leq 0,20$ na análise univariada e que, portanto, foram incluídas no modelo multivariado, cinco delas permaneceram no modelo final $(p \leq 0,10)$ : residir em domicílio com seis ou mais pessoas; possuir cuidador com menos de oito anos de estudo; frequentar pré-escola pública ou filantrópica; busca por serviços de saúde bucal ambulatorial e de emergência e ingestão diária de doces entre as refeições. Todas essas variáveis, com exceção da escolaridade do cuidador, apresentaram associação estatisticamente significante com o desfecho no modelo multivariado hierarquizado (Tabela 1). 
Tabela 1

Modelo de regressão logística para os fatores associados à prevalência de cárie dentária aos cinco anos de idade (ceod21). Análise multivariada hierarquizada. Recife, Pernambuco, Brasil, 2006.

\begin{tabular}{|c|c|c|c|c|c|c|}
\hline Variáveis/Categorias & $\begin{array}{c}\text { OR }_{\text {bruta }} \\
\text { OR [IC95\%] }\end{array}$ & $p$ & $\begin{array}{l}\text { OR } \text { ajustada1 }_{1} \\
\text { OR [IC95\%] }\end{array}$ & $p$ & $\begin{array}{l}\text { OR }_{\text {ajustada2 }} \\
\text { OR [IC95\%] }\end{array}$ & $p$ \\
\hline \multicolumn{7}{|l|}{ Nível distal } \\
\hline \multicolumn{7}{|l|}{ Fatores socioeconômicos } \\
\hline \multicolumn{6}{|l|}{ no domicílio } & 0,005 \\
\hline Até 3 & 1,00 & & 1,00 & & 1,00 & \\
\hline 4 a 5 & $2,31[1,40-3,79]$ & & $1,96[1,08-3,54]$ & & $1,78[0,92-3,45]$ & \\
\hline 6 ou mais & $4,56[2,62-7,95]$ & & $3,83[1,97-7,43]$ & & $3,35[1,60-7,02]$ & \\
\hline $\begin{array}{l}\text { Escolaridade materna } \\
\text { (anos de estudo) }\end{array}$ & & $<0,001$ & & 0,044 & & 0,182 \\
\hline$<8$ & $3,91[2,41-6,33]$ & & $2,02[0,86-4,76]$ & & $1,81[0,68-4,83]$ & \\
\hline $8-10$ & $2,23[1,28-3,86]$ & & $3,22[1,28-8,09]$ & & $2,59[0,94-7,14]$ & \\
\hline 11 ou mais & 1,00 & & 1,00 & & 1,00 & \\
\hline Ocupação do pai & & 0,080 & & 0,030 & & 0,126 \\
\hline Especializado & 1,00 & & 1,00 & & 1,00 & \\
\hline Semi-especializado & $0,64[0,20-2,23]$ & & $0,64[0,16-2,56]$ & & $0,57[0,13-2,43]$ & \\
\hline Não-especializado & $1,31[0,42-4,14]$ & & $1,52[0,40-5,78]$ & & $1,22[0,30-4,89]$ & \\
\hline Não ocupado & $0,97[0,29-3,25]$ & & $1,08[0,27-4,33]$ & & $0,90[0,21-3,83]$ & \\
\hline $\begin{array}{l}\text { Escolaridade do cuidador } \\
\text { (anos de estudo) }\end{array}$ & & $<0,001$ & & 0,052 & & 0,244 \\
\hline$<8$ & $4,26[2,52-7,21]$ & & $1,98[0,80-4,89]$ & & $1,73[0,62-4,75]$ & \\
\hline $8-10$ & $1,93[1,08-3,44]$ & & $0,80[0,31-2,05]$ & & $0,87[0,31-2,45]$ & \\
\hline 11 ou mais & 1,00 & & 1,00 & & 1,00 & \\
\hline \multicolumn{7}{|l|}{ Nível intermediário } \\
\hline \multicolumn{7}{|l|}{ Fatores do contexto de vida } \\
\hline Tipo de pré-escola & & $<0,001$ & & $<0,001$ & & 0,091 \\
\hline Privada & 1,00 & & 1,00 & & 1,00 & \\
\hline Pública & $2,37[1,54-3,64]$ & & $2,51[1,53-4,13]$ & & $1,69[0,93-3,07]$ & \\
\hline Filantrópica & $3,42[1,21-9,67]$ & & $4,21[1,08-16,42]$ & & $2,11[0,54-8,22]$ & \\
\hline Não frequenta & $1,37[0,80-2,35]$ & & $1,01[0,54-1,88]$ & & $0,80[0,39-1,65]$ & \\
\hline \multicolumn{7}{|l|}{ Razão da procura as USF } \\
\hline Tratamento & & $<0,001$ & & $<0,001$ & & $<0,001$ \\
\hline Sim & $4,81[2,59-8,95]$ & & $5,69[2,55-12,72]$ & & $4,23[1,93-9,28]$ & \\
\hline Não & 1,00 & & 1,00 & & 1,00 & \\
\hline Emergência & & 0,001 & & 0,046 & & 0,048 \\
\hline $\operatorname{sim}$ & $4,80[1,85-12,50]$ & & $3,20[1,02-10,08]$ & & $4,00[1,01-15,86]$ & \\
\hline Não & 1,00 & & 1,00 & & 1,00 & \\
\hline \multicolumn{7}{|l|}{$\begin{array}{l}\text { Fatores relativos a atitudes } \\
\text { de cuidado }\end{array}$} \\
\hline $\begin{array}{l}\text { Consumo de doces entre } \\
\text { as refeições }\end{array}$ & & $<0,001$ & & $<0,001$ & & $<0,001$ \\
\hline Não & 1,00 & & 1,00 & & 1,00 & \\
\hline Às vezes & $1,56[0,81-2,99]$ & & $1,47[0,76-2,8]$ & & $1,64[0,68-3,90]$ & \\
\hline Diariamente & $4,36[2,26-8,40]$ & & $4,22[2,17-8,18]$ & & $4,51[1,89-10,76]$ & \\
\hline
\end{tabular}

$\mathrm{OR}_{\text {bruta }}=$ análise univariada; $\mathrm{OR}_{\text {ajustada } 1}=$ ajustada pelas outra variáveis internamente ao bloco; $\mathrm{OR}_{\text {ajustada2 }}=\mathrm{ajustada}$ internamente ao bloco e pelo(s) blocos superiores; IC $95 \%=$ intervalo de $95 \%$ de confiança; OR=Odds ratio; Ajuste do modelo: -2 Log $L R=398,59$ e $R^{2}=0,35$. 
Modelo de regressão logística e fatores de risco associados à prevalência de cárie dentária aos cinco anos de idade (ceod-21). Análise multivariada convencional. Recife, Pernambuco, Brasil, 2006.

\begin{tabular}{|c|c|c|c|c|}
\hline Variáveis/Categorias & $\begin{array}{c}\text { OR }_{\text {bruta }} \\
\text { OR [IC95\%] }\end{array}$ & $p$ & $\begin{array}{c}\text { OR }_{\text {ajustada }} \\
\text { OR [IC95\%] }\end{array}$ & \\
\hline Número de moradores no domicílio & & $<0,001$ & & $<0,014$ \\
\hline Até 3 & 1,00 & & 1,00 & \\
\hline $4-5$ & $2,31[1,40-3,79]$ & & $1,68[0,86-3,30]$ & \\
\hline 6 ou mais & $4,56[2,62-7,95]$ & & $2,96[1,41-6,24]$ & \\
\hline $\begin{array}{l}\text { Escolaridade do cuidador (anos de } \\
\text { estudo) }\end{array}$ & & $<0,001$ & & 0,013 \\
\hline$<8$ & $4,26[2,52-7,21]$ & & $2,63[1,34-5,15]$ & \\
\hline $8-10$ & $1,93[1,08-3,44]$ & & $1,59[0,75-3,33]$ & \\
\hline 11 ou mais & 1,00 & & 1,00 & \\
\hline Tipo de pré-escola & & $<0,001$ & & 0,026 \\
\hline Privada & 1,00 & & 1,00 & \\
\hline Pública & $2,37[1,54-3,64]$ & & $2,11[1,17-3,79]$ & \\
\hline Filantrópica & $3,42[1,21-9,67]$ & & $2,20[0,56-8,61]$ & \\
\hline Não frequenta & $1,37[0,80-2,35]$ & & $0,93[0,46-1,87]$ & \\
\hline \multicolumn{5}{|l|}{ Razão da procura as USF } \\
\hline Tratamento & & $<0,001$ & & $<0,001$ \\
\hline Sim & $4,81[2,59-8,95]$ & & $4,07[1,85-8,95]$ & \\
\hline Não & 1,00 & & 1,00 & \\
\hline Emergência & & 0,001 & & 0,027 \\
\hline Sim & $4,81[1,85-12,50]$ & & $4,42[1,17-16,61]$ & \\
\hline Não & 1,00 & & 1,00 & \\
\hline Consumo de doces entre as refeições & & $<0,001$ & & $<0,001$ \\
\hline Não & 1,00 & & 1,00 & \\
\hline Às vezes & $1,56[0,81-2,99]$ & & $1,45[0,61-3,44]$ & \\
\hline Diariamente & $4,36[2,26-8,40]$ & & $4,64[1,95-11,01]$ & \\
\hline
\end{tabular}

$\mathrm{OR}_{\text {bruta }}=$ análise univariada; $\mathrm{OR}_{\text {ajustada }}=$ valores ajustados pelas outras variáveis; $I C 95 \%=$ intervalo de $95 \%$ de confiança; OR=odds ratio; Ajuste do modelo: -2 Log $L R=391,38$ e R2 $=0,34$.

\section{Discussão}

A comparação entre os resultados das análises dos dados utilizando dois modelos (hierarquizado e convencional) mostrou diferentes resultados na investigação dos determinantes da cárie nas crianças estudadas. Constatou-se que o modelo hierarquizado final incluiu um número maior de variáveis socioeconômicas, as quais estão situadas no nível distal de determinação, que foram mantidas no modelo conforme a metodologia proposta nesta abordagem, que recomenda a permanência das variáveis de primeiro nível no modelo final, independentemente do nível de significância estatística obtido. Diferentemente, quando as variáveis socioeconômicas foram ajustadas no modelo de regressão logística multivariado convencional, observou-se que um menor número delas permaneceu no modelo final, visto que as associações perderam significância estatística. Contudo, ao se comparar os resultados obtidos com os modelos de análise hierarquizada e convencional, constatou-se que os resultados finais obtidos foram de certo modo concordantes.

Entre as variáveis do bloco socioeconômico, verificou-se que a alta densidade de moradores no domicílio manteve-se fortemente associada ao desfecho, tanto no modelo multivariado convencional, como no modelo hierarquizado, mesmo quando essa variável foi ajustada duas vezes no modelo hierarquizado. Resultados semelhantes foram encontrados em estudo conduzido por Melo et al.,20 
ao utilizar o modelo de regressão logística multivariada convencional na análise dos dados deste estudo, assim como Gao et al.,21 quando usou modelos de equações estruturais, uma técnica estatística multivariada que permite avaliar simultaneamente as relações entre múltiplos construtos.

A baixa escolaridade do cuidador foi um fator de risco socioeconômico que esteve associado à cárie apenas no modelo multivariado convencional. A permanência desta variável no modelo hierarquizado final como um indicador de risco de primeiro nível, assim como a escolaridade materna e a ocupação do pai, só ocorreu por força da estruturação desse modelo, que não recomenda a retirada, das variáveis distais não significantes, nesta etapa da análise. ${ }^{11}$ Isto provocou, em relação às variáveis escolaridade da mãe e do cuidador, um efeito de inversão de risco entre as categorias da variável escolaridade materna (menos de oito anos de estudos: $\mathrm{OR}=1,81$; e entre oito e dez anos: $\mathrm{OR}=2,59$ ), quando o esperado é um maior risco de ter cárie entre crianças, cujas mães possuem os mais baixos níveis de escolaridade. Esse comportamento não ocorreu para a variável escolaridade do cuidador. Vale destacar que o percentual de cuidadores diários das crianças em sua maioria era constituído de mães (72,8\%). Já no modelo convencional, a escolaridade da mãe não foi significante e o efeito da escolaridade do cuidador expressa-se coerente, devido ao fato da maioria dos cuidadores ser constituída pelas mães das crianças. Ademais, apesar de se esperar a presença de colinearidade entre a escolaridade do cuidador e a escolaridade materna, o modelo multivariado para o nível distal indicou $\left(\mathrm{OR}_{\text {ajustada1 }}\right)$ serem, essas duas variáveis, fatores independentes associados ao desfecho com níveis de significâncias $p=0,05$ e $p=0,04$, respectivamente.

No modelo hierarquizado, os resultados obtidos para as variáveis de ordem socioeconômica foram concordantes com outros estudos que também empregaram análise hierarquizada dos dados. Em três estudos nacionais ${ }^{14-16}$ foi observado que, dentre as diversas variáveis testadas, a renda14-16 e a escolaridade materna 15,16 foram os principais determinantes de nível distal para cárie em crianças até seis anos de idade. Um estudo internacional, em crianças de 48 meses de idade, encontrou associação entre nível de renda baixo e cárie precoce. Entretanto, no modelo final de regressão logística hierarquizada esse fator não foi incluído por ter perdido significância. ${ }^{22}$

Entre as variáveis do bloco contexto de vida e familiar, apenas duas, relativas ao suporte social, mantiveram associação estatisticamente significante com a cárie nos dois modelos multivariados finais.
Resultados semelhantes foram observados quando se utilizou a análise multivariada convencional. ${ }^{20}$ Apesar da semelhança de resultados, vale considerar, que a sua interpretação, quando guiada pelo modelo hierarquizado, possibilita uma discussão mais contextualizada por favorecer a identificação de fatores, com níveis de determinação hierarquicamente superiores, que influenciaram os fatores de nível intermediário. Estes últimos por sua vez, mostraram-se associados ao desfecho - o tipo de escola e o motivo da procura pelos serviços odontológicos.

Concordâncias com os resultados de outros estudos que investigaram o efeito dos fatores situados no nível distal sobre variáveis de nível hierárquico inferior, como a qualidade das préescolas, e destas, sobre o perfil de saúde bucal infantil,23 foram encontradas neste estudo. Entretanto, Peres et al.16 observaram associação entre não estudar em pré-escola pública e a presença de cárie entre crianças, e, classificou essa variável como de nível de determinação proximal, que por sua vez seria mediada por fatores situados em níveis de determinação distais, como a baixa escolaridade materna e renda familiar. Por outro lado, concordâncias com outros estudos sobre o uso de serviços odontológicos foram encontradas com estudos que utilizaram análise de regressão logística multivariada convencional.20,24,25

Sobre os resultados encontrados na análise do efeito das variáveis relativas às atitudes de cuidado, apenas o padrão de consumo diário de doces foi um fator que permaneceu associado à cárie nos dois modelos finais, ressaltando-se que essa variável, que foi classificada como de nível proximal na análise hierarquizada, foi a única cuja associação se manteve independentemente do efeito das variáveis pertencentes aos níveis de determinação superiores. Esses resultados mostram a elevada força de associação entre este fator com a cárie dentária nesta população quando comparada às outras explorações analíticas realizadas. ${ }^{26,27} \mathrm{Em}$ ambas as abordagens, as crianças com tal tipo de hábito apresentaram aproximadamente, quatro vezes mais chances de ter cárie, quando comparadas àquelas sem esse hábito. Ao serem realizadas comparações entre esses resultados com o de outras pesquisas verificou-se no estudo de Melo et al., ${ }^{20}$ que utilizou análise de regressão logística multivariada convencional, as chances de as crianças terem cárie foram menores assim como no estudo de Peres et al.,16 quando utilizou a análise de regressão logística hierarquizada.

Ressalta-se, no entanto, que a definição das 
preferências dietéticas é complexa e precocemente determinada, estando as escolhas alimentares fortemente relacionadas a fatores socioeconômicos e vinculados aos interesses da cadeia tecnológica agroindustrial do açúcar e seus derivados, com impacto negativo na saúde bucal e sistêmica das populações, devendo essa questão ser enfrentada na perspectiva da segurança alimentar. ${ }^{28}$ Apesar, dos resultados obtidos entre os modelos hierarquizado e convencional, serem semelhantes, a análise hierarquizada utilizada contribui para lançar luz, nessa discussão, pelo fato de ter incluído no seu modelo final a ocupação do pai e da escolaridade da mãe em razão do seu pressuposto teórico - apontando para uma discussão mais contextualizada referente a influência desses fatores sociais sobre esse padrão de consumo de açúcar das crianças e deste sobre o agravo em questão.

As principais limitações deste trabalho referemse ao desenho de estudo transversal, do qual os dados foram retirados e que impedem inferências causais, além da possibilidade de ocorrência de vieses de informação e de memória, devido ao elevado percentual de respondentes com baixa escolaridade. 19,20 Deve-se, também, considerar a limitação dos dois métodos de análise multivariados empregados com relação a possibilidade de apreender a complexidade do processo estudado, dado que apenas variáveis atribuídas ao nível do indivíduo foram consideradas.

Contudo, a inclusão da abordagem hierarquizada proposta por Victora et al. ${ }^{11}$ possibilitou evidenciar um maior número de fatores como indicadores de risco socioeconômico para o desfecho. Tal estratégia, menos sintética que a do modelo multivariado convencional, representou um avanço por permitir considerações relativas à ordenação temporal ou lógica entre as variáveis e hipóteses de intermediação de efeitos sobre o desfecho estudado. ${ }^{5,11}$ Entretanto, a abordagem por níveis apresenta limitações metodológicas relativas a não-estimação dos efeitos indiretos, representação limitada das inter-relações entre os níveis hierárquicos e a não obtenção de índices globais de ajuste. 11,12 Deve ser dada atenção às generalizações dos resultados. A população estudada é específica e oriunda de áreas urbanas pobres sob a influência das políticas de saúde da ESF do Recife, sendo pertinente comparação com populações semelhantes.

$\mathrm{Na}$ atualidade, no contexto de análises hierarquizadas, no qual as observações não podem ser consideradas independentes, outras abordagens analíticas vêm sendo indicadas, como os modelos multinível e de equações estruturais. ${ }^{5}$ Os modelos multinível por possibilitarem a análise de situações que contêm variáveis de diferentes níveis permitem identificar a variabilidade atribuída a cada nível na determinação pela introdução de efeitos aleatórios. 5,13 Já os modelos de equações estruturais, além de produzirem estimativas de efeitos indiretos em análises envolvendo variáveis latentes ou constructos, permitem lidar simultaneamente, e, com mais eficiência, com um número elevado de variáveis de diferentes domínios, fornecendo estimativas dos efeitos diretos e indiretos e promovendo a avaliação de mecanismos mediadores por meio da decomposição dos efeitos.5,21

Apesar das limitações, as abordagens analíticas exploradas reafirmaram a influência das condições precárias de vida na produção da cárie nas crianças estudadas. 20 A modelagem hierarquizada possibilitou identificar os determinantes de nível distal, que exerceram influências sobre aqueles de níveis inferiores e destes sobre o desfecho, favorecendo uma interpretação dos resultados, à luz do conhecimento existente, sobre as múltiplas dimensões do problema estudado. Além disso, possibilita o planejamento de ações de saúde, segundo a hierarquia dos efeitos das variáveis sobre o desfecho.

Assim, conclui-se que os dados deste estudo reforçam a superioridade dos modelos hierquizados de análise na produção de evidências para a formulação de políticas de saúde integrais e integradas voltadas à redução da cárie dentária na infância.

\section{Agradecimentos}

Os autores gostariam de agradecer a Secretaria de Saúde do Recife, a Pós-graduação de odontologia da UFPE e ao CPqAM/Fiocruz-PE pela parceria na viabilização deste estudo. E, as crianças e aos seus responsáveis por autorizarem os exames e entrevistas, que serviram de base para as análises deste estudo.

\section{Referências}

1. Bernabe E, Hobdell MH. Is income inequality related to childhood dental caries in rich countries? J Am Dent Assoc. 2010; 141: 143-9.
2. Antunes JLF, Narvai PC. Políticas de saúde bucal no Brasil e seu impacto sobre as desigualdades em saúde. Rev Saúde Pública. 2010; 44: 360-5. 
3. Brasil. Ministério da Saúde. Projeto SB Brasil 2010 Pesquisa Nacional de Saúde Bucal: resultados principais. Brasília, DF; 2010.

4. Terreri ALM, Soler ZASG. Estudo comparativo de dois critérios utilizados no Programa Saúde da Família na priorização do tratamento da cárie entre crianças de 5 a 12 anos. Cad Saúde Pública. 2008; 24: 1581-7.

5. Aleksejūniene J, Holst D, Brukiene V. Dental caries risk studies revisited: causal approaches needed for future inquiries. Int J Environ Res Public Health. 2009; 6 (12) 2992-3009.

6. Holst D,Schuller AA, Aleksejuniené J, Eriksen HM. Caries in populations - a theoretical, causal approach. Eur J Oral Sci. 2001; 109: 143-8.

7. Brunner E, Marmot M. Social organization, stress and health. In: Marmot M, Wilkinson R, organizadores. Socia determinants of health. Oxford: Oxford University; 1999. p. 17-43.

8. Dahlgren G, Whitehead M. Policies and strategies to promote social equity in health. Copenhagen: WHO, Regional Office for Europe; 1992.

9. Peres MA, Peres KG, Antunes JLF, Junqueira SR, Frazão P, Narvai PC. The association between socioeconomic development at the town level and distribution of dental caries in brazilian children. Rev Panam Salud Pública. 2003; 14: 149-57.

10. Mello TRC, Antunes JLF, Waldman EA. Prevalência de cárie não tratada na dentição decídua em áreas urbanas e rurais do Estado de São Paulo, Brasil. Rev Panam Salud Pública. 2008; 23: 78-84

11. Victora C, Huttly S, Fuchs S, Olinto M. The role of conceptual frameworks in epidemiological analysis: a hierarchical approach. Int J Epidemiol. 1997; 26: 224-7.

12. Fuchs SC, Victora CG, Fachel J. Modelo hierarquizado: uma proposta de modelagem aplicada à investigação de fatores de risco para diarréia grave. Rev Saúde Pública. 1996; 30: 168-78.

13. Bramlett MD, Soobader MJ, Fisher-Owens SA, Weintraub JA, Gansky SA, Platt LJ,Newacheck PW. Assessing a multilevel model of young children's oral health with national survey data. Community Dent Oral Epidemiol. 2010; 38: 287-98.

14. Catani DB, Cypriano S, Sousa MLR. Determinantes clínicos e sócio-comportamentais da cárie dentária nas dentições decídua e permanente em município com concentração ótima de fluoreto na água. Arq Odontol. 2010; 46: 197-207.

15. Oliveira LB, Sheiham A, Bönecker MJS. Exploring the association of dental caries with social factors and nutritional status in Brazilian preschool children. Eur J Oral Sci. 2008; 116: 37-43.

16. Marco Aurélio Peres, Maria do Rosário D. O. Latorre, AubreySheiham, Karen Glazer Peres, Fernando Celso Barros, Pedro Gonzales Hernandez, Angela Maria Nunes
Maas, Ana Regina Romano, Cesar Gomes Victora. Determinantes sociais e biológicos da cárie dentária em crianças de 6 anos de idade: um estudo transversal aninhado numa coorte de nascidos vivos no sul do Brasil. Rev Bras Epidemiol. 2003; 6: 293-6.

17. Almeida TF, Vianna MI, Cabral MB, Cangussu MC, Floriano FR. Family context and incidence of dental caries in preschool children living in areas covered by the Family Health Strategy in Salvador, Bahia State, Brazil. Cad Saúde Pública. 2012; 28: 1183-95.

18. Almeida TF, Cangussu MCT, Chaves SCL, Silva DIC, Santos SC. Condições de saúde bucal de crianças na faixa etária pré-escolar, residentes em áreas de abrangência do Programa Saúde da Família em Salvador, Bahia, Brasil. Rev Bras Saúde Matern Infant. 2009; 9: 247-52.

19. Melo MMDC, Souza WV, Lima MLC, Couto GBL, Chaves TM. Cárie dentária em pré-escolares: um estudo em áreas cobertas pela estratégia saúde da família do Recife, PE, Brasil. Pesq Bras Odontoped Clin Integr. 2010; 10: 337-43.

20. Melo MMDC, Souza WV, Lima MLC, Braga C. Fatores associados à cárie dentária em pré-escolares do Recife, Pernambuco, Brasil. Cad Saúde Pública. 2011; 27: 471-85.

21. Gao XL, Hsu CY, Xu YJ, Loh T, Koh D, Hwang HB. Behavioral Pathways Explaining Oral Health Disparity. J Dent Res. 2010: 89: 985-90.

22. Werneck RI, Lawrence HP, Kulkarni GV, Locker D. Early childhood caries and access to dental care among children of Portuguese-speaking immigrants in the city of Toronto. $\mathrm{J}$ Can Dent Assoc. 2008; 74 (9): 805.

23. Moysés ST, Moysés SJ, Watt RG, Sheiham A. Associations between health promoting schools policies and indicators of oral health in Brazil. Health Promot Int. 2003; 18: 20918

24. Pine CM, Adair PM, Burnside G, Nicoll AD, Gillett A, Borges-Yáñez SA, Broukal Z, Brown J, Declerck D, Ping FX, Gugushe T, Hunsrisakhun J, Lo EC, Naidoo S,Nyandindi U, Poulsen VJ, Razanamihaja N, Splieth C, Sutton BK, Soo TC. Barriers to the treatment of childhood caries perceived by dentists working in different countries. Community Dent Health. 2004; 21 (Suppl. 1): 112-20.

25. Mantonanaki M, Koletsi-Kounari H, Mamai-Homata E, Papaioannou W. Prevalence of dental caries in 5-year-old Greek children and the use of dental services: evaluation of socioeconomic, behavioural factors and living conditions. Int Dent J. 2013; 63: 72-9.

26. Wigen TI, Wang NJ. Caries and background factors in Norwegian and immigrant 5-year-old children. Community Dent Oral Epidemiol. 2010; 38 (1): 19-28.

27. Mobley C, Marshall TA, Milgrom P, Coldwell SE. The contribution of dietary factors to dental caries and disparities in caries. Acad Pediatr. 2009; 9: 410-4.

28. Custódio MB, Furquim NR, Santos GMM, Cyrillo DC. Segurança alimentar e nutricional e a construção de sua política: uma visão histórica. Segur Alimentar Nutr. 2011; 18 (11): $1-10$.

Recebido em 26 de dezembro de 2013

Versão final apresentada em 19 de maio de 2014

Aprovado em 25 de junho de 2014 